\title{
Protein Science
}

\section{The crystal structure of the E. coli stress protein YciF}

Aditya Hindupur, Deqian Liu, Yonghong Zhao, Henry D. Bellamy, Mark A. White and Robert O. Fox

Protein Sci. published online Sep 25, 2006;

Access the most recent version at doi:10.1110/ps.062307706

$\mathbf{P}<\mathbf{P} \quad$ Published online September 25, 2006 in advance of the print journal.

Email alerting Receive free email alerts when new articles cite this article - sign up in the box at the service top right corner of the article or click here

Notes

Advance online articles have been peer reviewed and accepted for publication but have not yet appeared in the paper journal (edited, typeset versions may be posted when available prior to final publication). Advance online articles are citable and establish publication priority; they are indexed by PubMed from initial publication. Citations to Advance online articles must include the digital object identifier (DOIs) and date of initial publication.

To subscribe to Protein Science go to:

http://www.proteinscience.org/subscriptions/ 


\title{
The crystal structure of the E. coli stress protein YciF
}

\author{
ADITYA HINDUPUR,,${ }^{1,4}$ DEQIAN LIU, ${ }^{1,4}$ YONGHONG ZHAO, ${ }^{1,3}$ HENRY D. BELLAMY, ${ }^{2}$ \\ MARK A. WHITE, ${ }^{1}$ AND ROBERT O. FOX ${ }^{1}$ \\ ${ }^{1}$ Department of Biochemistry and Molecular Biology and The Sealy Center for Structural Biology \& Molecular \\ Biophysics, The University of Texas Medical Branch, Galveston, Texas 77555-0647, USA \\ ${ }^{2}$ Center for Advanced Microstructures and Devices, Louisiana State University, Baton Rouge, Louisiana 70806, USA
}

(Received April 27, 2006; Final Revision July 19, 2006; Accepted July 20, 2006)

\begin{abstract}
YciF is a protein that is up-regulated when bacteria experience stress conditions, and is highly conserved in a range of bacterial species. YciF has no known structure or biochemical function. To learn more about its potential molecular function and its role in the bacterial stress response, we solved the crystal structure of YciF at $2.0 \AA$ resolution by the multiple wavelength anomalous diffraction (MAD) technique. YciF is a dimer in solution, and forms a homodimer in the crystal asymmetric unit. The two monomers form a dimer with a molecular twofold axis, with a significant burial of solvent-accessible surface area. The protein is an all-alpha protein composed of five helices: a four-helix bundle, and a short additional helix at the dimer interface. The protein is structurally similar to portions of the diiron-containing proteins, rubrerythrin and the Bacillus anthracis Dlp-2.
\end{abstract}

Bacterial responses to stress conditions include the production of the relatively well-characterized heat-shock response and responses to osmotic or acid stress. The heat-shock proteins (HSPs) include molecular chaperones (GroEL, DnaK, DnaJ), and ATP-dependent proteases, including Lon and ClpA. YciF is one such protein that has been identified as being produced by bacteria in response to stress conditions. It is encoded by the yciF gene (Blattner et al. 1997), and is predicted to be the second gene in the yciGFE-katN operon (Robbe-Saule et al. 2001). The YciF homolog in Salmonella enterica appears to be under the control of RpoS, with a putative RpoS promoter upstream of yciG (Ibanez-Ruiz et al. 2000). YciF expression was up-regulated 20 -fold in response to hyperosmolarity, and approximately threefold upon entering stationary phase or after acid shock at $\mathrm{pH} 5$.

\footnotetext{
${ }^{3}$ Present address: Department of Pharmacology, The University of Texas Medical Branch, Galveston, TX 77555-1031, USA.

${ }^{4}$ These authors contributed equally to this work.

Reprint requests to: Robert O. Fox, Department of Biochemistry and Molecular Biology, University of Texas Medical Branch, 301 University Blvd., Mail Route 0647, Galveston, TX 77555-0647, USA; e-mail: fox@bloch.utmb.edu; fax: (409) 747-4745.

Article published online ahead of print. Article and publication date are at http://www.proteinscience.org/cgi/doi/10.1110/ps.062307706.
}

Later work on the same species indicated that yciGFEkatN is regulated by bile, independent of RpoS control (Prouty et al. 2004). A proteomics study carried out on the phytopathogen Xanthomonas axonopodis revealed expression of a YciF homolog when exposed to host leaf extract (Tahara et al. 2003).

The Escherichia coli yciF gene encodes a 166 amino acid protein $(18,597.1 \mathrm{Da})$ with a calculated isoelectric point of 5.47. The function of YciF is unknown, and although it is conserved across bacterial species, it has no close homologs whose biological function or structure is known.

\section{Results and Discussion}

\section{Twinning}

We reported earlier that YciF crystals occurred in the R32 space group (Liu et al. 2004). Due to the difficulties encountered while building a model for an MIR solution (data not shown), the possibility of merohedral twinning was considered. Statistical analysis of the diffraction data for possible twinning (Yeates 1997) revealed that the crystals were in space group R3 with nearly perfect merohedral twinning, and had a twin fraction $\sim 0.5$, which led to the apparent R32 space group. The twinning 
was eliminated by purifying and crystallizing the protein in the presence of EDTA (see Materials and Methods). The untwinned crystals occur in the space group R3, and have a $V_{\mathrm{m}}$ of $2.5 \AA^{3} / \mathrm{Da}$ calculated with two molecules in the asymmetric unit.

\section{Monomer structure}

The structure was solved by multiple wavelength anomalous diffraction (MAD) and refined to $2.0 \AA$ resolution with a final $R_{\text {work }}$ and $R_{\text {free }}$ of $18.3 \%$ and $22.4 \%$, respectively. The crystal was found to have two identical chains in the asymmetric unit (Chains A and B). The map displayed clear electron density for 158 residues in Chain A (3-161) and 153 residues (4-157 residues) in Chain $\mathrm{B}$, and 118 ordered water molecules. The first two and the last five residues in Chain A are disordered, as are the first three and last nine residues in the B chain; no electron density was observed for these regions. The two molecules of the asymmetric unit were found to be nearly identical in their core structure $(\mathrm{C} \alpha$ RMSD, $0.309 \AA$ for residues 5-150 excluding loop residues 85-100), but to differ in this loop, as judged by an overall C $\alpha$ RMSD of $0.729 \AA$. The crystallographic data are summarized in Table 1.

The overall secondary structure of the protein (Fig. 1) was found to be composed of $5 \alpha$-helices: H1 (6-33), H2

Table 1. Data collection and refinement statistics

\begin{tabular}{|c|c|c|c|}
\hline $\begin{array}{l}\text { MAD data } \\
\text { collection and processing }\end{array}$ & Inflection & Peak & Remote \\
\hline \multicolumn{4}{|l|}{ Data set } \\
\hline Wavelength $(\AA)$ & 0.9794 & 0.9797 & 0.9252 \\
\hline Resolution range $(\AA)$ & $50-1.90$ & $50-1.90$ & $50-1.90$ \\
\hline Unique reflections & 24,356 & 24,693 & 29216 \\
\hline Measured reflections & 43,281 & 47,576 & 52,819 \\
\hline Completeness $(\%)$ & 88.9 & 96.3 & 90.4 \\
\hline$R_{\mathrm{sym}}(\%)$ & $5.9(26.1)$ & $6.6(24.1)$ & $71(35.1)$ \\
\hline$I / \sigma(I)$ & 9.28 & 14.84 & 11.76 \\
\hline \multicolumn{4}{|l|}{ Phasing statistics } \\
\hline Resolution range $(\AA)$ & $20-1.9$ & & \\
\hline Se sites/monomer & 4 & & \\
\hline $\mathrm{FOM}_{\mathrm{MAD}}$ & 0.47 & & \\
\hline FOM $_{\text {RESOLVE }}$ & 0.66 & & \\
\hline \multicolumn{4}{|l|}{ Model refinement } \\
\hline Resolution range $(\AA)$ & 2.00 & & \\
\hline No. of reflections & 19,178 & & \\
\hline No. of protein atoms & 2461 & & \\
\hline No. of water molecules & 118 & & \\
\hline$R_{\text {work }}(\%)$ & 18.3 & & \\
\hline$R_{\text {free }}(\%)$ & 22.4 & & \\
\hline \multicolumn{4}{|l|}{ Stereochemistry } \\
\hline RMSD for bond length $(\AA)$ & 0.007 & & \\
\hline RMSD for bond angles $\left({ }^{\circ}\right)$ & 1.2 & & \\
\hline \multicolumn{4}{|l|}{ Residues in the Ramachandran plot } \\
\hline Most favored region (\%) & 97.0 & & \\
\hline Additional allowed regions (\%) & 2.3 & & \\
\hline Generously allowed regions (\%) & 0.7 & & \\
\hline
\end{tabular}
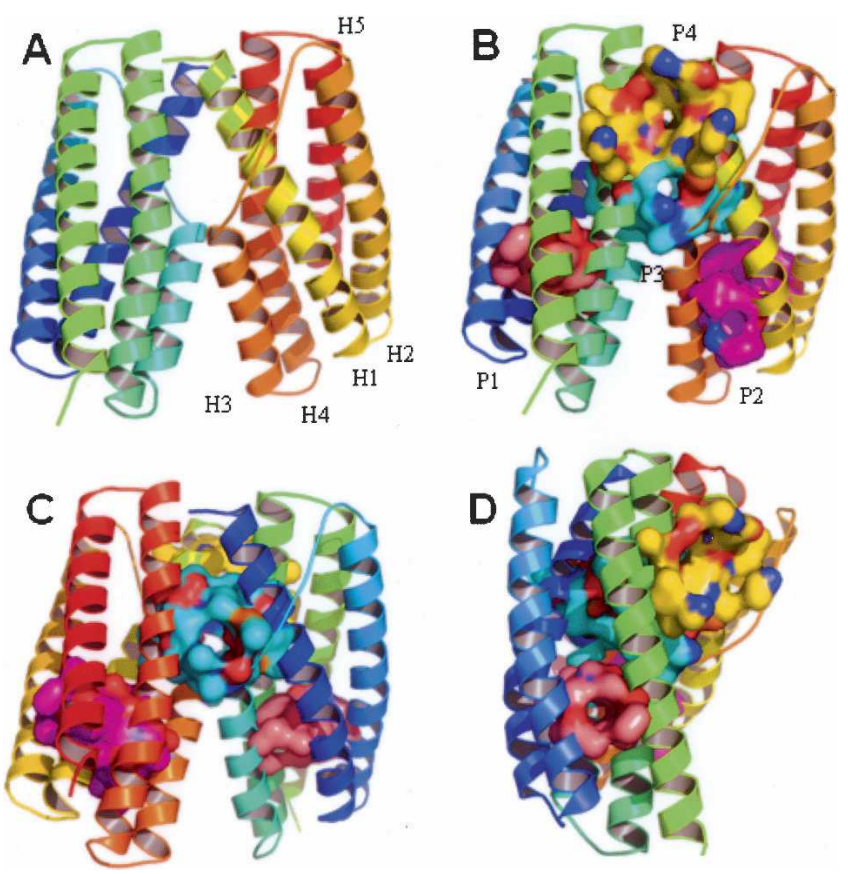

Figure 1. (A) The dimer structure of YciF. $(B-D)$ The potential ligandbinding regions of the protein. In $A$ and $B$ the molecular twofold axis is vertical in the plane of the paper; in $C$ and $D$ the view is rotated by $180^{\circ}$ and $90^{\circ}$, respectively, along the vertical twofold axis relative to $A$. The surfaces of the potential ligand-binding pockets are labeled, P1 (brown), P2 (magenta), P3 (cyan), and P4 (yellow).

(38-63), H3 (76-89), H4 (96-125), and H5 (129-159). The shortest helix (H3) is connected to $\mathrm{H} 2$ by a 12 amino acid-long loop. The overall protein fold has been classified as DUF892 (family of several hypothetical bacterial proteins of no known function) by PFAM (v17.0) (Bateman et al. 2004). This fold is shared by at least 57 other proteins in the database. The loop region between $\mathrm{H} 3$ and $\mathrm{H} 4$ may adopt an ensemble of conformations; this is evident from the temperature factors of the atoms in this loop. The average B-factor for the atoms in the loop H3-H4 is 40.38 $\AA^{2}$ for chain $\mathrm{A}$, whereas in chain $\mathrm{B}$ it has an average $\mathrm{B}$-factor of $20.55 \AA^{2}$.

\section{Dimer interface}

YciF forms a dimer in solution, as demonstrated by sizeexclusion chromatography. The crystal packing presents two potential dimer interfaces, each with significant buried surface area. The first solution dimer model has a local molecular twofold axis and a buried solventaccessible surface area of $2143 \AA^{2}$. This protein dimer is relatively compact, with dimensions of $45 \times 50 \times 25 \AA$. The dimer interface involves three helices from each monomer (H1, H3, and $\left.\mathrm{H} 4, \mathrm{H}^{\prime}, \mathrm{H}^{\prime}{ }^{\prime}, \mathrm{H} 4{ }^{\prime}\right)$; salt bridges between Lys108-Glu79 and Lys4-Asp8, and hydrogen 
bonds between Gln126-Lys4 and Lys74-His111, would stabilize this interaction. Of the interacting residues in the first dimer model, Lys108, Glu79, and Gln126 are conserved in half of the sequences that were aligned, and two residues, Lys4 and Asp8, are conserved in more than half of the sequences. The second solution dimer model involves an interface between two antiparallel helices, $\mathrm{H} 2-\mathrm{H} 2{ }^{\prime}$. In this model a local molecular twofold parallel to the c-axis of the unit cell would relate the two monomers. This dimer interface would be stabilized by just four hydrogen bonds, with a buried surface area of only $1257 \AA^{2}$. Based on these observations, we propose that the first solution dimer model described is the biologically relevant structure (Fig. 1).

\section{Analysis of potential ligand-binding sites}

Analysis of the YciF structure with CASTp (Liang et al. 1998; Binkowski et al. 2003) allows visualization of the pockets present on the protein surface. There are four distinct pockets per dimer, with P1 and P2 occurring within the core of the four-helix bundles and related by a molecular twofold axis. P1 has a solvent-accessible (SA) area of $62 \AA^{2}$ and a solvent-accessible volume (SAV) of 11 $\AA^{3}$, while P2 has an SA of $99 \AA^{2}$ and an SAV of $17 \AA^{3}$. The numerical and visual differences in P1 and P2 (Fig. 1) indicate that strict twofold symmetry is not obeyed for all side chains of the dimer. The third and largest pocket P3 (SA $352 \AA^{2}$, SAV $164 \AA^{3}$ ) forms at the dimer interface; a fourth, P4 (SA $154 \AA^{2}$, SAV $186 \AA^{3}$ ) lies just below P3, with its opening aligned with the entrance of $\mathrm{P} 3$.

Pockets P3 and P4 occur near, and are related by the twofold axis, and have potential ligand- or metal-binding sites involving Asp16, Cys75, and Tyr122' from the opposite monomer. Additionally, the pockets are lined with polar residues: His12, and His111' from the opposite monomer, and the pocket shapes differ (Fig. 1). An analysis of the sequence alignment with other bacterial homologs shows that His111, Cys75, and Asp16 are conserved across all species, and that His 12 is conserved in 10 out of the 15 homologs (Fig. 2), suggesting conservation of the pocket. The overall appearance of the pocket suggests a binding site for an ion and/or a small molecule.

\section{Structural comparisons}

The YciF protein is highly conserved across the Enterobacteriaciae members, with sequence identities ranging from $72 \%-86 \%$. The identity between $\mathrm{YciF}$ and its orthologs in Xanthomonas, Bordetalla, and Nitrobacter is 55\%, 54\%, and 48\%, respectively (Altschul et al. 1990). YciE, another member of the yciGFE-katN operon, has $21 \%$ identity and $41 \%$ similarity (over 144 amino acid residues) with YciF, and can be detected by using PSI-BLAST (Altschul et al. 1997).

A homology search with DALI reveals the closest matches to be rubrerythrin from Desulfovibrio vulgaris (1RYT; RMSD $2.1 \AA$ for $134 \mathrm{C} \alpha$ atoms; deMare et al. 1996), a non-heme iron-containing ferritin from Listeria innocua (1QGH; RMSD $2.2 \AA$ for $137 \mathrm{C} \alpha$ atoms; Ilari et al. 2000), bacterioferritin from Escherichia coli (1BCF; RMSD $2.2 \AA$ for $139 \mathrm{C} \alpha$ atoms; Frolow et al. 1994), and a DNA-binding protein from Halobacterium salinarum (1MOJ, RMSD $2.7 \AA$ for $144 \mathrm{C} \alpha$ atoms; Zeth et al. 2004). The sequence identity with these proteins was found to be between $7 \%$ and $16 \%$. A similarity search of the YciF monomer structure against PDB entries using the Molecular Structure Database gave Dlp-2, another iron-binding protein from Bacillus anthracis, as the top hit, with an RMSD of $1.71 \AA$ for 126 residues of chain B matched to 146 residues of the Dlp-2 chain B (1JIG:B; Papinutto et al. 2002). The other top hit was Listeria innocua Dps chain D, RMSD $1.74 \AA$, for 128 residues chain B of YciF, matched to 150 residues chain D of Dps (2BJY: D; Ilari et al. 2005). Both Dlp-2 and Dps are iron-binding proteins with similar monomer folds: four-helix bundles with a short additional helix that occurs within the connecting loop between the second and third long helices. Similarities between YciF and Dlp-2 or Dps are limited only to the monomer fold, and do not extend to the overall architecture of the molecules. Metal binding in Dps family members occurs at dimer interfaces, while metal binding by YciF is proposed to occur within the monomeric four-helix bundle.

\section{Rubrerythrin and sulerythrin}

Rubrerythrin ( $\mathrm{Rr}$ ) was initially isolated from the anaerobic Desulfovibrio vulgaris (deMare et al. 1996). The archeon Pyrococcus furiosus rubrerythrin has peroxidase activity in vitro (Weinberg et al. 2004), but pyrophosphatase (Liu and Le Gall 1990), ferroxidase (Bonomi et al. 1996), and superoxide dismutase (Lehmann et al. 1996) activities have also been reported. The $\mathrm{Rr}$ monomer consists of two domains: one, an N-terminal four-helix bundle containing a diiron site similar to hemerythrin, and the other, a C-terminal rubredoxin-like domain with an $\mathrm{Fe}(\mathrm{SCys})_{4}$ site (deMare et al. 1996; Jin et al. 2002; $2004 a, b)$. The two irons are coordinated by five carboxylates and a single His, and are bridged by an oxo ion. Nigerythrin, a rubrerythrin-like protein, shows a $2.0 \AA$ movement of $\mathrm{Fe} 1$ from a carboxylate to a histidine residue when converted from the mixed valency Fe2(II)-Fe1(III) state to a diferrous state, similar to that seen in $\mathrm{Rr}$, indicating that this is a characteristic feature of the Rr-like diiron proteins (Iyer et al. 2005). Sulerythrin is the first Rr-like protein to be isolated from an 

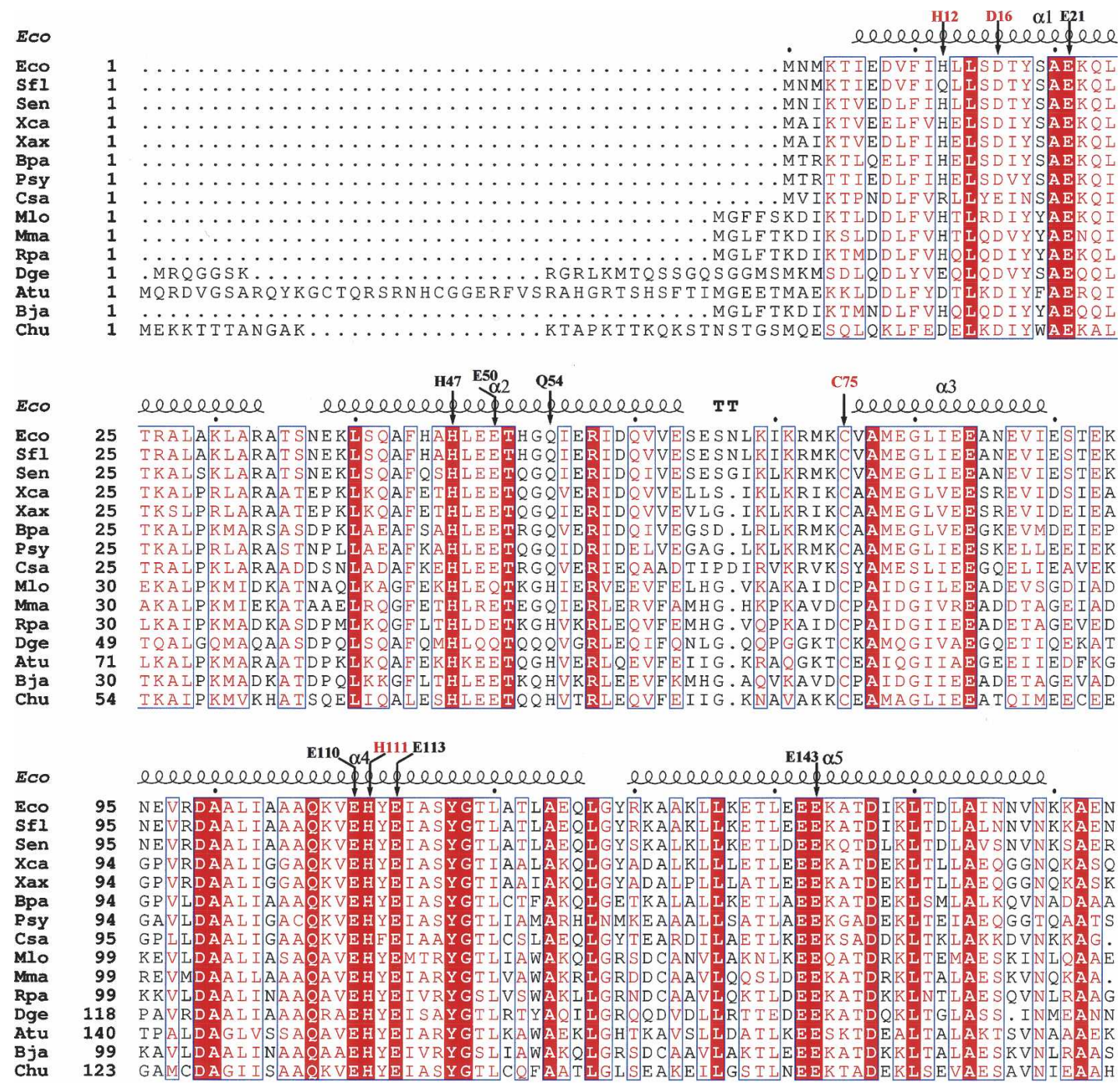

Figure 2. Alignment of YciF with its homologs identified from PFAM (v17.0). The secondary structure assignments are taken from the crystal structure. Abbreviations are: Eco, Escherichia coli; Sfl, Shigella flexneri; Sen, Salmonella enterica; Xca, Xanthomonas campestris; Xax, Xanthomonas axonopodis; Bpa, Bordetella parapertusis; Psy, Pseudomonas syringae; Csa, Chromohalobacter salexigens; Mlo, Mesorhizobium loti; Mma, Magnetospirillum magnetotacticum; Rpa, Rhodopseudomonas palustris; Dge, Deinococcus geothermalis; Atu, Agrobacterium tumefaciens; Bja, Bradyrhizobium japonicum; and Chu, Cytophaga hutchinsonii. The alignment was generated using ClustalW, and the image was generated using the Espript server. The conserved residues that occur in the P1 and P2 sites are identified by black lettering: E21, H47, E50, Q54, E110, E113, and E143. The conserved residues that occur in the P3 and P4 sites are identified by red lettering: H12, D16, C75, H111, and Y112 (not labeled for clarity).

aerobe, Sulfolobus tokadaii strain 7. The protein is a homodimer that lacks the C-terminal rubredoxin-like domain and shows binuclear metal binding of $\mathrm{Fe} / \mathrm{Zn}$ (Fushinobu et al. 2003).

\section{Similarity of YciF to rubrerythrin and sulerythrin}

In an alignment of rubrerythrin with YciF, the four helices 1,2, 4, and 5 overlap clearly with those of the rubrerythrin four-helix bundle (Fig. 3). Alignment of YciF with rubrerythrin places the diiron center of rubrerythrin in the middle of the YciF monomer. One of these metal ions (M1) is placed to be well coordinated by Glu110, His47, and Glu50. The second metal ion (M2) would be coordinated by YciF residues Glu21, Glu113, and Gln54. Glu143 is in a position to serve as a bridging residue between the two metal sites. A structure-based alignment of the YciF and Rr sequences shows conservation of residues at positions Glu20:Glu21 (Rr:YciF), Glu94:Glu110, Glu97:Glu113, and Glu128:Glu143. Sulerythrin has a similar coordination of its diiron center within a four-helix bundle (Fushinobu et al. 2003; Wakagi 2003). Both YciF and sulerythrin lack a rubredoxin 


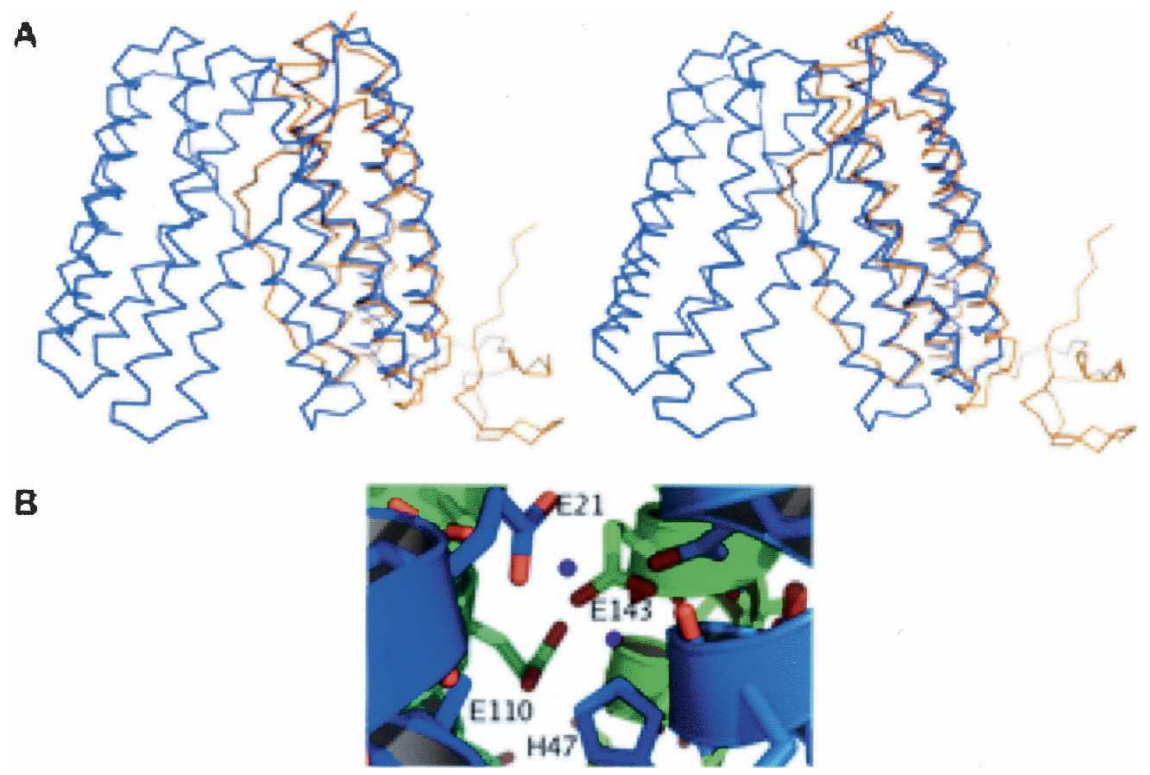

Figure 3. (A) Structural alignment of YciF (cyan) and rubrerythrin (red). (B) The Rr Fe atoms (magenta) were placed into the YciF structure by superposition of similar Rr and YciF C $\alpha$ atoms. Side chains (visible in the figure) that could bind a diiron center are labeled. The positions of the side chains are as seen in the crystal structure; no attempt has been made to reorient them to model the potential binding site. The two N-terminal helices are in blue, and the two C-terminal helices are in green.

domain. Based on these observations, YciF appears to be a member of the Rr family.

$\mathrm{X}$-ray fluorescence spectra were measured around the Fe $\mathrm{K}$ absorption edge to test for the presence of iron. The spectra were taken from a buffer control $(20 \mathrm{mM}$ Tris, 50 $\mathrm{mM} \mathrm{NaCl}, 5 \mathrm{mM} \mathrm{BME}$ at pH 7.8), YciF (10 mg/mL), and YciF-Fe $(10 \mathrm{mg} / \mathrm{mL})$. The YciF-Fe protein was prepared as described in Materials and Methods. The YciF-Fe solution showed a small but significant absorption edge around 7125 $\mathrm{eV}$, whereas the other solutions did not. Spectrophotometric analysis of the protein solution revealed a broad absorption band centered at $522 \mathrm{~nm}$, a slightly longer wavelength than that seen for chopped $\operatorname{Rr}(474 \mathrm{~nm})$ containing only the diiron site (Gupta et al. 1995). The X-ray fluorescence data and optical spectrum suggest that YciF may possess a diiron site similar to its structural homolog rubrerythrin.

\section{Summary and conclusions}

YciF is a protein that is up-regulated when the bacteria encounter stress conditions. Based on the structural comparison of YciF to rubrerythrin, a protein that has a diiron center, and to ferritin and other monooxygenases that are all known to be metal binding, we hypothesize that YciF is a metal-binding protein. The metal-binding sites are proposed to lie in the helix bundle within the two pockets (P1 and P2), which are related by a molecular twofold axis of symmetry, with Glu110, Glu50, and His47 coordinating one metal ion and Glu113, Gln54, and Glu21 coordinating the second ion. Also, Glu143 may act as a bridging residue akin to Glu128 in Rr. The architectural features of YciF are also found associated with other protein functions, such as, the manganese catalase domain in the protein bll3758 from Bradyrhizobium japonicum, suggesting a functional role in protecting the cell against oxidative damage.

\section{Materials and methods}

\section{Protein preparation and crystallization}

Protein preparation and crystallization were as published earlier (Liu et al. 2004), with the following modification to eliminate merohedral twinning. The protein was purified in the presence of $10 \mathrm{mM}$ EDTA following purification on the nickel column. Treating with EDTA resulted in the formation of crystals in the R3 space group, instead of R32, as reported previously. In brief, the proteins were crystallized in $0.1 \mathrm{M}$ citric acid ( $\mathrm{pH} 3.5-$ 4.0), 1.9-2.1 M ammonium sulfate, and $10 \mathrm{mM}$ EDTA. The crystals were step-soaked into the cryoprotectant $(0.1 \mathrm{M}$ citric acid at $\mathrm{pH} 3.5,2.0 \mathrm{M}$ ammonium sulfate, and $30 \%[\mathrm{v} / \mathrm{v}]$ glycerol) prior to flash freezing in $\mathrm{LN}_{2}$. To explore the potential incorporation of $\mathrm{Fe}$ into the protein structure, $\mathrm{FeSO}_{4}(3 \mathrm{mM}$ final concentration) was added at the time of induction with IPTG, and the protein was prepared as above, but without the addition of EDTA.

\section{Structure determination and refinement}

Flash-frozen selenomethionine-labeled YciF crystals were used for data collection. The data sets were collected at the Protein Crystallography MAD beamline at CAMD using a marCCD 
detector. The data were collected at three wavelengths corresponding to inflection, peak, and remote wavelengths for Se. The images were indexed, integrated, and scaled using HKL2000 (Otwinowski and Minor 1997). The space group was R3, with cell dimensions $a=b=79.96 \AA, c=131.12 \AA, \alpha=\beta=90^{\circ}, \gamma=120^{\circ}$. There were two molecules per asymmetric unit, giving a Matthew's coefficient of $2.23 \AA^{3} / \mathrm{Da}$, which corresponds to a solvent content of $45 \%$ (Matthews 1968).

SOLVE (Terwilliger and Berendzen 1999) was used to find the selenium sites and to calculate the phases. A total of four selenium sites were found. The initial figure of merit (FOM) before solvent flattening was 0.43. Solvent flattening and automated model building were carried out with RESOLVE (Terwilliger 2000); solvent flattening improved the FOM to 0.66 . RESOLVE built $\sim 70 \%$ of the model. Model building and corrections were done using Xfit (McRee 1999). The waters were added manually with Xfit, and using PMB/CNS. The structure refined to a final $R_{\text {work }}$ of $18.3 \%$ and an $R_{\text {free }}$ of $22.4 \%$, within a resolution range of $50-2.0 \AA$, using the programs PMB (Scott et al. 2004) and CNS (Brunger et al. 1998). The model quality was checked using the PROCHECK (Laskowski et al. 1993) in the CCP4 suite (Collaborative Computational Project 1994). The refined model had $96 \%$ of its residues in the most favored region of the Ramachandran Plot, $3.7 \%$ in the allowed region, and one residue in the disallowed region (ChainA:Asn95); reexamination of the structure very clearly showed the ChainA:Asn95 to be well positioned within the electron density. GETAREA (Fraczkiewicz and Braun 1998) was used to calculate the solvent-accessible surface area. The molecular surface and electrostatic potential were calculated using SwissPDB.

\section{PDB accession numbers}

The coordinates and structure factors have been deposited in the Protein Data Bank (accession code 2GS4).

\section{Acknowledgments}

This work was supported by a grant from The Welch Foundation (H-1345 to ROF), with Center support from The Sealy and Smith Foundation. We gratefully acknowledge support from the NSF through the biological infrastructure award DBI-9871464, which provided the construction funds for the beamline used in these studies, with interagency matching funds from the NIGMS at the $\mathrm{NIH}$ and the GCPCC member institutions. H.D.B. is partially supported through the Louisiana Governor's Biotechnology Initiative. We thank Dr. David Konkel for editing the manuscript.

\section{References}

Altschul, S.F., Gish, W., Miller, W., Myers, E.W., and Lipman, D.J. 1990. Basic local alignment search tool. J. Mol. Biol. 215: 403-410.

Altschul, S.F., Madden, T.L., Schaffer, A.A., Zhang, J., Zhang, Z., Miller, W., and Lipman, D.J. 1997. Gapped BLAST and PSI-BLAST: A new generation of protein database search programs. Nucleic Acids Res. 25: 3389-3402.

Bateman, A., Coin, L., Durbin, R., Finn, R.D., Hollich, V., Griffiths-Jones, S., Khanna, A., Marshall, M., Moxon, S., Sonnhammer, E.L., et al. 2004 The Pfam protein families database. Nucleic Acids Res. 32: D138 D141.

Binkowski, T.A., Naghibzadeh, S., and Liang, J. 2003. CASTp: Computed atlas of surface topography of proteins. Nucleic Acids Res. 31: 3352-3355.
Blattner, F.R., Plunkett 3rd, G., Bloch, C.A., Perna, N.T., Burland, V., Riley, M., Collado-Vides, J., Glasner, J.D., Rode, C.K., Mayhew, G.F., et al. 1997. The complete genome sequence of Escherichia coli K-12. Science 277: 14531474 .

Bonomi, F., Kurtz, D.M.J., and Cui, X. 1996. Ferroxidase activity of recombinant Desulfovibrio vulgaris rubrerythrin. J. Biol. Inorg. Chem. 1: $67-72$.

Brunger, A.T., Adams, P.D., Clore, G.M., DeLano, W.L., Gros, P., GrosseKunstleve, R.W., Jiang, J.S., Kuszewski, J., Nilges, M., Pannu, N.S., et al. 1998. Crystallography \& NMR system: A new software suite for macromolecular structure determination. Acta Crystallogr. D Biol. Crystallogr. 54: $905-921$.

Collaborative Computational Project, Number 4. 1994. The CCP4 suite: Programs for protein crystallography. Acta Crystallogr. D Biol. Crystallogr. 50: $760-763$.

deMare, F., Kurtz Jr., D.M., and Nordlund, P. 1996. The structure of Desulfovibrio vulgaris rubrerythrin reveals a unique combination of rubredoxin-like FeS4 and ferritin-like diiron domains. Nat. Struct. Biol. 3: 539-546.

Fraczkiewicz, R.B. and Braun, W. 1998. Exact and efficient analytical calculation of the accessible surface areas and their gradients for macromolecules. J. Comput. Chem. 19: 319-333.

Frolow, F., Kalb, A.J., and Yariv, J. 1994. Structure of a unique twofold symmetric haem-binding site. Nat. Struct. Biol. 1: 453-460.

Fushinobu, S., Shoun, H., and Wakagi, T. 2003. Crystal structure of sulerythrin, a rubrerythrin-like protein from a strictly aerobic archaeon, Sulfolobus tokodaii strain 7, shows unexpected domain swapping. Biochemistry 42: $11707-11715$.

Gupta, N., Bonomi, F., Kurtz Jr., D.M., Ravi, N., Wang, D.L., and Huynh, B.H. 1995. Recombinant Desulfovibrio vulgaris rubrerythrin. Isolation and characterization of the diiron domain. Biochemistry 34: 3310-3318.

Ibanez-Ruiz, M., Robbe-Saule, V., Hermant, D., Labrude, S., and Norel, F. 2000. Identification of RpoS $\left(\sigma^{\mathrm{s}}\right)$-regulated genes in Salmonella enterica serovar Typhimurium. J. Bacteriol. 182: 5749-5756.

Ilari, A., Stefanini, S., Chiancone, E., and Tsernoglou, D. 2000. The dodecameric ferritin from Listeria innocua contains a novel intersubunit iron-binding site. Nat. Struct. Biol. 7: 38-43.

Ilari, A., Latella, M.C., Ceci, P., Ribacchi, F., Su, M., Giangiacomo, L., Stefanini, S., Chasteen, N.D., and Chiancone, E. 2005. The unusual intersubunit ferroxidase center of Listeria innocua Dps is required for hydrogen peroxide detoxification but not for iron uptake. A study with sitespecific mutants. Biochemistry 44: 5579-5587.

Iyer, R.B., Silaghi-Dumitrescu, R., Kurtz Jr., D.M., and Lanzilotta, W.N. 2005 High-resolution crystal structures of Desulfovibrio vulgaris (Hildenborough) nigerythrin: Facile, redox-dependent iron movement, domain interface variability, and peroxidase activity in the rubrerythrins. J. Biol. Inorg. Chem. 10: 407-416.

Jin, S., Kurtz Jr., D.M., Liu, Z.J., Rose, J., and Wang, B.C. 2002. X-ray crystal structures of reduced rubrerythrin and its azide adduct: A structure-based mechanism for a non-heme diiron peroxidase. J. Am. Chem. Soc. 124: 9845-9855.

. 2004a. Displacement of iron by zinc at the diiron site of Desulfovibrio vulgaris rubrerythrin: X-ray crystal structure and anomalous scattering analysis. J. Inorg. Biochem. 98: 786-796.

2004b. X-ray crystal structure of Desulfovibrio vulgaris rubrerythrin with zinc substituted into the $\left[\mathrm{Fe}(\mathrm{SCys})_{4}\right]$ site and alternative diiron site structures. Biochemistry 43: 3204-3213.

Laskowski, R.A., MacArthur, M.W., Moss, D.S., and Thorton, J.M. 1993. PROCHECK: A program to check the stereochemical quality of protein structures. J. Appl. Crystallogr. 26: 283-291.

Lehmann, Y., Meile, L., and Teuber, M. 1996. Rubrerythrin from Clostridium perfringens: Cloning of the gene, purification of the protein, and characterization of its superoxide dismutase function. J. Bacteriol. 178: 7152 7158.

Liang, J., Edelsbrunner, H., and Woodward, C. 1998. Anatomy of protein pockets and cavities: Measurement of binding site geometry and implications for ligand design. Protein Sci. 7: 1884-1897.

Liu, D., Zhao, Y., Fan, X., Sun, Y., and Fox, R.O. 2004. Escherichia coli stress protein YciF: Expression, crystallization and preliminary crystallographic analysis. Acta Crystallogr. D Biol. Crystallogr. 60: 2389-2390.

Liu, M.Y. and Le Gall, J. 1990. Purification and characterization of two proteins with inorganic pyrophosphatase activity from Desulfovibrio vulgaris: Rubrerythrin and a new, highly active, enzyme. Biochem. Biophys. Res. Commun. 171: 313-318.

Matthews, B.W. 1968. Solvent content of protein crystals. J. Mol. Biol. 33: 491-497. 
McRee, D.E. 1999. XtalView/Xfit-A versatile program for manipulating atomic coordinates and electron density. J. Struct. Biol. 125: 156 165 .

Otwinowski, Z. and Minor, W. 1997. Processing of X-ray diffraction data collected in the oscillation mode, pp. 307-326. Academic Press, New York

Papinutto, E., Dundon, W.G., Pitulis, N., Battistutta, R., Montecucco, C., and Zanotti, G. 2002. Structure of two iron-binding proteins from Bacillus anthracis. J. Biol. Chem. 277: 15093-15098.

Prouty, A.M., Brodsky, I.E., Manos, J., Belas, R., Falkow, S., and Gunn, J.S. 2004. Transcriptional regulation of Salmonella enterica serovar Typhimurium genes by bile. FEMS Immunol. Med. Microbiol. 41: 177-185.

Robbe-Saule, V., Coynault, C., Ibanez-Ruiz, M., Hermant, D., and Norel, F. 2001. Identification of a non-haem catalase in Salmonella and its regulation by $\operatorname{RpoS}\left(\sigma^{\mathrm{S}}\right)$. Mol. Microbiol. 39: 1533-1545.

Scott, E.E., White, M.A., He, Y.A., Johnson, E.F., Stout, C.D., and Halpert, J.R. 2004. Structure of mammalian cytochrome P450 2B4 complexed with 4-(4chlorophenyl)imidazole at $1.9-\AA$ resolution: Insight into the range of P450 conformations and the coordination of redox partner binding. J. Biol. Chem. 279: 27294-27301
Tahara, S.T., Mehta, A., and Rosato, Y.B. 2003. Proteins induced by Xanthomonas axonopodis pv. passiflorae with leaf extract of the host plant (Passiflorae edulis). Proteomics 3: 95-102.

Terwilliger, T.C. 2000. Maximum-likelihood density modification. Acta Crystallogr. D Biol. Crystallogr. 56: 965-972.

Terwilliger, T.C. and Berendzen, J. 1999. Automated MAD and MIR structure solution. Acta Crystallogr. D Biol. Crystallogr. 55: 849-861.

Wakagi, T. 2003. Sulerythrin, the smallest member of the rubrerythrin family, from a strictly aerobic and thermoacidophilic archaeon. Sulfolobus tokodaii strain 7. FEMS Microbiol. Lett. 222: 33-37.

Weinberg, M.V., Jenney Jr., F.E., Cui, X., and Adams, M.W. 2004. Rubrerythrin from the hyperthermophilic archaeon Pyrococcus furiosus is a rubredoxindependent, iron-containing peroxidase. J. Bacteriol. 186: 7888-7895.

Yeates, T.O. 1997. Detecting and overcoming crystal twinning. Methods Enzymol. 276: 344-358.

Zeth, K., Offermann, S., Essen, L.O., and Oesterhelt, D. 2004. Iron-oxo clusters biomineralizing on protein surfaces: Structural analysis of Halobacterium salinarum DpsA in its low- and high-iron states. Proc. Natl. Acad. Sci. 101: 13780-13785. 